\title{
Site-Specific Polarization Screening in Organic Thin Films
}

\author{
S. Soubatch, C. Weiss, R. Temirov, and F. S. Tautz* \\ JARA-Fundamentals of Future Information Technology and Institut für Bio- und Nanosysteme 3, \\ Forschungszentrum Jülich, 52425, Germany \\ (Received 19 November 2008; published 1 May 2009)
}

\begin{abstract}
Systematic local spectroscopy of the affinity levels, by means of a scanning tunneling microscope, in highly ordered molecular semiconductor films of tetracene reveals strong energy level shifts by up to $\sim 1.0 \mathrm{eV}$ from molecule to molecule. This final state effect can be traced back to the site specificity of the polarization energy in organic materials with complex unit cells, caused by a combination of different molecular environments, the intrinsically anisotropic molecular polarizability, and the influence of the substrate.
\end{abstract}

PACS numbers: 31.70.Ks, 68.37.Ef, 78.55.Kz

Organic semiconductor materials attract considerable attention, the reason being their relevance for electronic and optoelectronic devices [1-3]. Because of their importance for device functions, the electronic properties of molecules close to interfaces have been studied in particular [4]. For example, it has been shown that the chemical bonding to a metal surface may change both the electronic levels and geometric structure of organic semiconductor molecules profoundly [5-9]. During charge transport, the electronic states of a molecular material may also be influenced by polarization screening, i.e., the stabilization of a locally injected charge through the polarization of the surrounding molecular environment [10,11]. While the principles of polaron-formation are well understood, the new aspect which we report here, namely, the site specificity of the polarization energy in complex unit cells and at surfaces of organic materials, was not experimentally proven so far. In fact, up to now attempts to measure directly the expected influence of local molecular environments on the polarization screening have failed $[12,13]$, for reasons which are currently not understood.

To clarify this issue, we systematically analyze the molecular energy levels of a suitable model system, a highly ordered but structurally complex organic semiconductor film which we have investigated by local scanning tunneling spectroscopy (STS) in situ at $8 \mathrm{~K}$ in a vacuum better than $10^{-10}$ mbar. We indeed find a remarkable site specificity of the polarization screening, in addition to the well-known influence by metallic substrate $[14,15]$. This influence reveals itself through a surprisingly large dispersity of the affinity level from molecule to molecule. Our findings have implications for transport in (bulk) organic semiconductors, because even chemically homogeneous materials may under certain circumstances be considered to consist of electronically distinct species (with an energy level dispersity up to $0.5 \mathrm{eV}$ ), with profound consequences for carrier transfer from molecule to molecule, since carriers may get trapped not only at defects but also at regular lattice sites. The results also make clear why under certain circumstances it may be difficult to observe the influence of intermolecular polarization screening in spectroscopic experiments $[12,13]$.

The experiments have been carried out on the $\beta$ phase of tetracene/ $\operatorname{Ag}(111)$. Tetracene is a platelet molecule for which high charge carrier mobilities have been demonstrated $[16,17]$. It was evaporated from an effusion cell at $420 \mathrm{~K}$ onto the silver surface at $210 \mathrm{~K}$, where-depending on the preparation procedure-it may form two long-range ordered phases ( $\alpha$ and $\beta$ ) [18]. At a nominal coverage of two monolayers (calibrated with respect to the compact $\alpha$-phase monolayer [18]) regions of both $\alpha$ and $\beta$ phases coexist on the surface (Fig. 1). The structure of the $\alpha$ phase as derived from our STM images (flat-lying molecules, unit cell vectors $b_{1}=8.2 \AA, b_{2}=13.1 \AA, \gamma=93^{\circ}$ ) agrees with the diffraction study reported previously [18]. The intramolecular contrast at positive bias voltages [Fig. 1(a), inset) resembles the geometry of lowest unoccupied molecular orbital (LUMO).

The $\beta$ phase consists of two structural motifs, a longrange domain lattice which is visible in Fig. 1 and the local crystal structure discernible in Figs. 2(a) and 2(b). Figure 2(c) displays the structural model derived from the STM data [19]. The $\beta$ phase is an ideal object for studying polarization screening in varying molecular environments, since it contains seven structurally nonequivalent types of

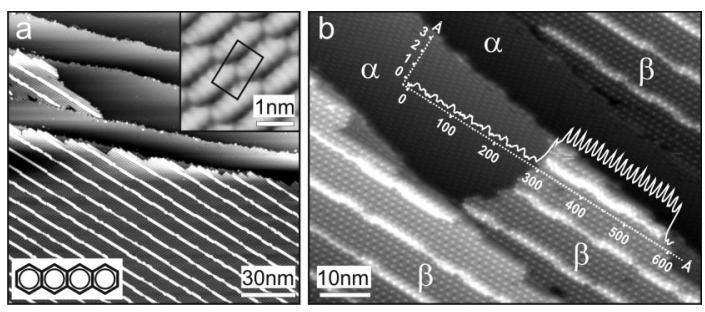

FIG. 1. STM images of $\alpha$ and $\beta$ phases of tetracene/ $\operatorname{Ag}(111)$. Tunneling parameters: (a) $I_{t}=25 \mathrm{pA}, U_{\text {bias }}=1.9 \mathrm{~V}$, (b) $I_{t}=38 \mathrm{pA}, U_{\text {bias }}=2 \mathrm{~V}$. Insets: Submolecular resolution of the $\alpha$ phase $\left(I_{t}=0.1 \mathrm{nA}, U_{\text {bias }}=1.5 \mathrm{~V}\right)$ and structure formula of tetracene. 

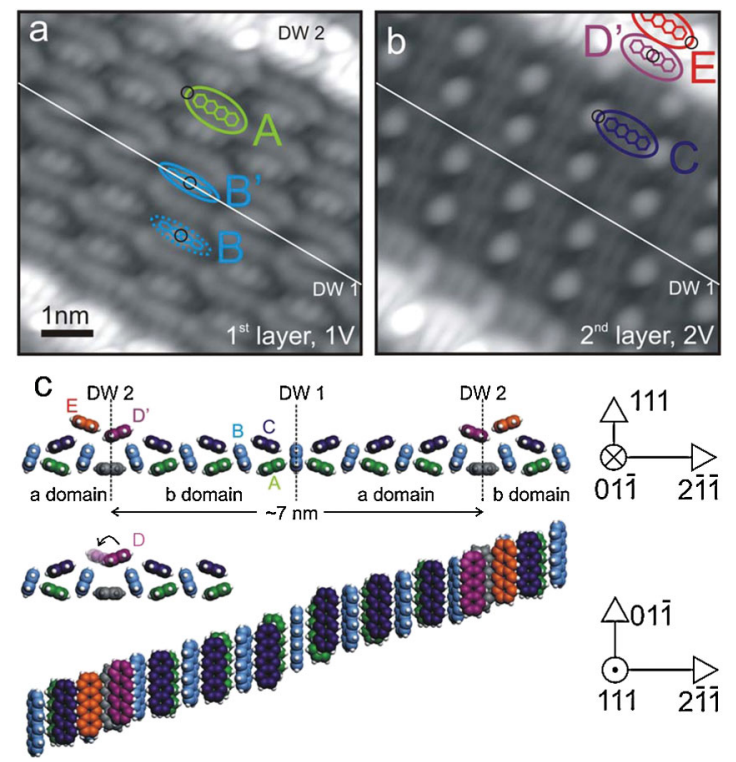

FIG. 2 (color). STM images (tunneling current $47 \mathrm{pA}$ ) of $\beta$ phase recorded at different voltages with molecule types marked: (a) $1.0 \mathrm{~V}$, (b) $2.0 \mathrm{~V}$. Black circles are positions where spectra of Fig. 3(b) have been measured. (c) Schematic side and top views (upper and lower models, respectively) of the $\beta$-phase structure. Molecules are colored as in (a) and (b) and labeled accordingly. Gray molecules are not visible in STM images. Domains and domain walls as defined in main text are indicated. The two equivalent positions of $D$ molecules in DW2 are also shown.

tetracene molecules, all of which are individually accessible to STS.

It is clear that the local crystal structure of the $\beta$ phase resembles the bulk structure of tetracene with its characteristic herringbone arrangement of molecules which are tilted around their long axis with respect to each other. The $\beta$ phase is a two-layer structure [19]. The first layer consists of $A$ and $B$ molecules. Since its overall area density of 1.5 molecules $/ \mathrm{nm}^{2}$ is larger than for a compact layer of flat molecules, we must conclude that either $A$ or $B$ molecules are tilted out of the surface plane. A careful analysis of the data shows that $B$ molecules are more strongly tilted (e.g., cf. the line shape of the LUMO spectra discussed below). $C$ molecules in the second layer are oriented nearly parallel to the substrate because they exhibit a similar footprint as molecules in the $\alpha$ phase and a comparable submolecular structure.

The long-range domain lattice consists of alternating domains for which the local crystal structure is rotated by $180^{\circ}$ around the surface normal. This implies the existence of two types of domain walls which are marked DW1 and DW2 in Fig. 2(c). The domain wall spacing is approximately $7 \mathrm{~nm}$. Across DW1 walls, we mainly observe an offset between adjacent rows of $C$ molecules. Moreover, symmetry requires that $B$ molecules in DW1 must have their $y$ axes [cf. Fig. 3(b), upper right inset] strictly parallel to the surface normal; we label these special molecules $B^{\prime}$. DW2 walls are decorated by molecular chains which are
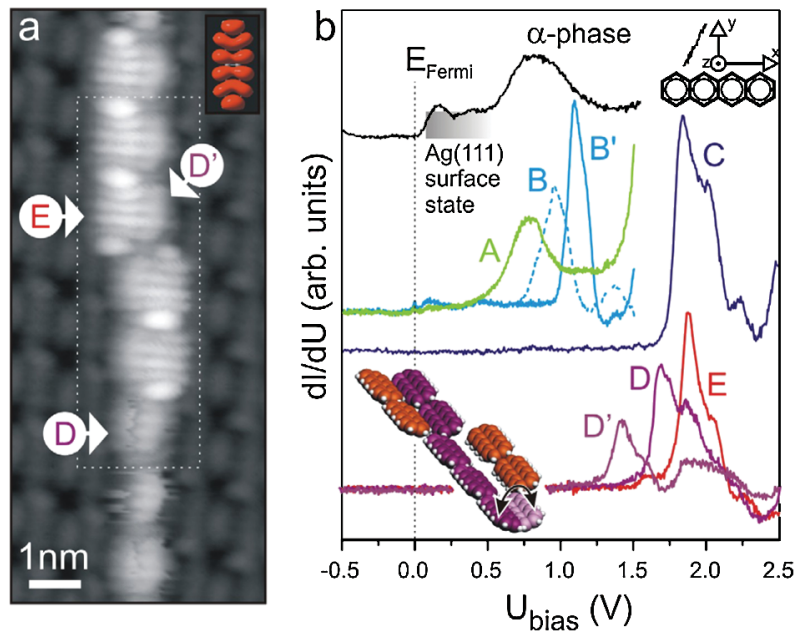

FIG. 3 (color). (a) STM image of DW2 with chain $\left(I_{t}=\right.$ $30 \mathrm{pA}, U_{\text {bias }}=2.24 \mathrm{~V}$ ). Molecules $D, E$, and $D^{\prime}$ are marked. Inset: Calculated tetracene LUMO. (b) Tunneling spectra of different tetracene molecules: $\alpha$ phase (black); $\beta$ phase: colors as in Fig. 2. Lower left inset: 3D model of the tetracene chain atop DW2 [dashed box in (a)]. Upper right inset: Molecular axes of tetracene.

oriented along the $[01 \overline{1}]$ direction [Fig. 3(a)]. The nature and origin of the chains, which consist of two types of molecules, follow from the structure of the domains themselves: $B$ molecules on either side of DW2 are tilted towards each other. This reduces the space available for second layer $C$ molecules in DW2 (which we call $D$ molecules from now on), with the result that in DW2 these are pushed slightly upwards [hence their brighter contrast in Figs. 2(a) and 2(b)]. At the same time, depending on their tilt, $D$ molecules can either be part of the $a$ or $b$ domains. This means that separate $D$ molecules can switch between two positions (and corresponding tilts) [Fig. 2(c)]. This bistability can indeed be observed in Fig. 3(a) as a streaky pattern in the STM image (lower part of the chain). If a second molecule, the $E$ molecule, binds next to it, the $D$ molecule is stabilized in one of the two configurations. It is then called a $D^{\prime}$ molecule. Apparently, the activation barrier between the two possible orientations of the $E-D^{\prime}$ dimer is larger, such that a switching action of the dimer is never observed under present experimental conditions. Altogether, the structure of the domain walls implies the existence of four additional types of molecules: $B^{\prime}, D, D^{\prime}$, and $E$, in addition to $A, B$, and $C$ molecules in the domains.

We turn to the electronic properties of our model system and demonstrate the site specificity of the polarization energy in the $\beta$ phase of tetracene. The most striking observation is the energy dispersity of the LUMO, with $E_{A}=0.8 \mathrm{eV}, \quad E_{B}=0.95 \mathrm{eV}, \quad E_{B^{\prime}}=1.10 \mathrm{eV}, \quad E_{D^{\prime}}=$ $1.42 \mathrm{eV}, \quad E_{D}=1.68 \mathrm{eV}, \quad E_{C}=1.84 \mathrm{eV}, \quad$ and $E_{E}=$ $1.87 \mathrm{eV}$ [20]. Since STS is sensitive to final state effects [21], contributions of polarization screening must be present in the spectra of Fig. 3(b). Tetracene has an anisotropic polarizability, because the delocalized charge den- 
sity in the $\pi$ orbitals is more easily displaced within the molecular plane than perpendicular to it. An $a b$ initio calculation (GAUSSIAN 03, BPW91/cc-pVDZ) [22] yields diagonal electronic polarizability tensor elements $\alpha_{x x}=$ $7.47 \times 10^{-39} \mathrm{C} \mathrm{m}^{2} / \mathrm{V}, \quad \alpha_{y y}=3.38 \times 10^{-39} \mathrm{C} \mathrm{m}^{2} / \mathrm{V}$, $\alpha_{z z}=1.05 \times 10^{-39} \mathrm{Cm}^{2} / \mathrm{V}$.

We will argue now that the site-specific screening of injected charges by surrounding molecules (and by the metal) is in fact the origin of the observed energy level dispersity. To this end, let us first consider a hypothetical tetracene dimer [Fig. 4(a)]. The two molecules of the pair are structurally nonequivalent, because the left (right) molecule, respectively, points its $y$ axis $(z$ axis) at its partner molecule. The figure illustrates that under these circumstances charges on either of these two molecules will be screened to different extents, and thus different affinity levels must be expected for the two molecules. Generalizing this result, we observe that the two constitutive elements of the situation in Fig. 4(a), namely, distinct environments of neighboring molecules and the anisotropy of the molecular polarizability, are also realized in the crystal structure of the $\beta$ phase of tetracene and can indeed-in conjunction with the substrate influence-explain the main features of the energy level sequence, as the results of a microelectrostatic model calculation to be discussed now show [Fig. 4(b)].
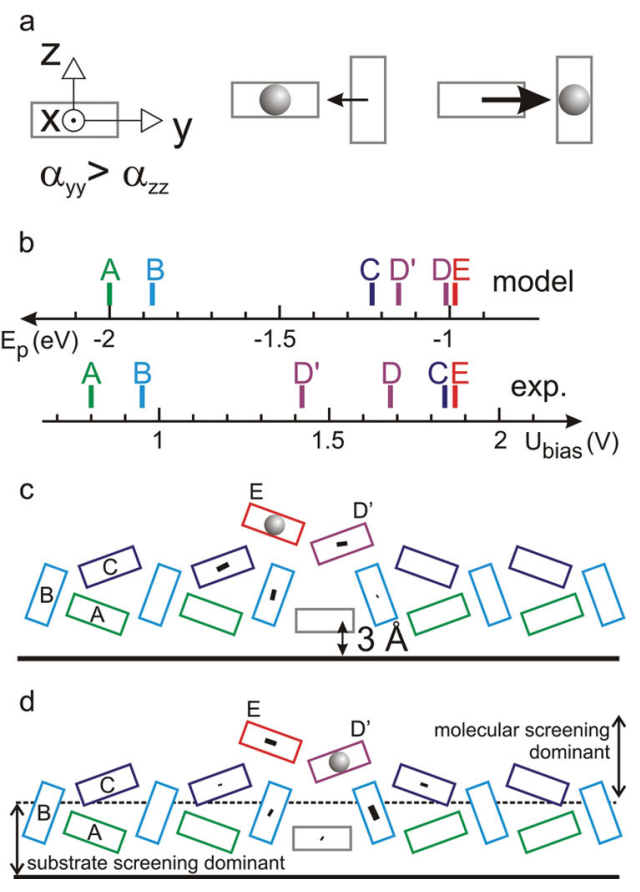

FIG. 4 (color). (a) Hypothetical tetracene dimer (side view). Sphere indicates injected electron, arrows the polarization response of the other molecule. (b) LUMO energy spectrum of tetracene $\beta$ phase, model as described in text (top) and experimental spectra of Fig. 3 (bottom). Spectra have been aligned at the $E$ molecule. (c),(d) $\beta$ phase side view with electron injected into $E$ (c) or $D^{\prime}$ (d). The thicknesses and lengths of the bars in adjacent molecules scale with the induced dipoles.
In the microelectrostatic model calculation [10], a single electron is first placed on a defined molecule within the $\beta$-phase structure, then the induced dipoles on the neighboring molecules are evaluated and the electrostatic attraction between the initial charge and the induced dipoles is calculated using simple analytic expressions for point charges and point dipoles. This model is an accurate description of the STS experiment, because at typical currents the time lapse between successively injected electrons is longer than the localization time of the electron, and hence single electrons are injected in our STS experiment into specified molecules. The screening of the metal is included at the level of image charge attraction [14]. The microelectrostatic model calculation is based on the structure in Fig. 2(c), with an assumed minimal metalmolecule distance of $3 \AA$, which is a reasonable estimate [7]. We further presume that the lifetime of the injected charge on all molecules is large enough for the neighboring molecules to fully polarize. Since electronic polarization occurs on the femtosecond scale [10], this is a safe assumption.

Following the principles illustrated by Fig. 4(a), the calculated sequence in Fig. 4(b) can be rationalized. Let us first concentrate on molecules $D, D^{\prime}$, and $E$ for which the intermolecular screening is dominant. Figures 4(c) and 4(d) show schematic plots of the polarization induced in adjacent molecules when a charge is injected into either a $D^{\prime}$ or $E$ molecule of the film. The comparison immediately explains the inequality $E_{E}>E_{D^{\prime}}$ as an outcome of the polarizability anisotropy: Efficient screening of the charge on $E$ would require the easy axis $\left(\alpha_{y y}>\alpha_{z z}\right)$ of the nearest neighbors $C, D^{\prime}$, and $B$ to point towards $E$ [23]. However, the $y$ axes of these molecules and consequently their induced dipoles (especially of $C$ and $D^{\prime}$ which are closest to $D$ ) are tilted away from this direction. In contrast, the easy axes of molecules $E, B$, and $C$ point towards $D^{\prime}$ and thus screen charges on $D^{\prime}$ effectively. Furthermore, we note that $E_{D^{\prime}}<E_{D}$ is correctly predicted; evidently this indicates that the screening influence of $E$ on $D^{\prime}$ is described realistically. We note that, in spite of yielding a correct overall tendency, the model underestimates (overestimates) the screening of $D, D^{\prime}$ molecules ( $C$ molecules). For $D$ and $D^{\prime}$ this could be a consequence of small deviations of the correct structure from the one in Fig. 2(c), while for $C$ this indicates a limitation of the model: The various screening contributions are split into pair interactions and added up. This approximation apparently breaks down for $C$ molecules, for which in reality the screening by the metal is substantially reduced by the interjacent $A$ molecules, yielding a larger LUMO energy in experiment. Moreover, it must be remembered that our model takes neither the inescapable intramolecular delocalization of the injected and screening charges nor higher order polarization effects into account. This would require a full selfconsistent $a b$ initio calculation of electronic structure in the presence of the injected charge. 
For molecules in the first monolayer of the $\beta$ phase the screening influence of the metal dominates. The sequence $E_{A}<E_{B}<E_{B^{\prime}}$ follows naturally from the increasing distance (angle) of the respective molecule from (to) the metal. Note that on the basis of the metal screening alone the difference $E_{B}-E_{A}$ would be much larger $(0.36 \mathrm{eV}$ instead of $0.15 \mathrm{eV}$ in experiment), but the better intermolecular screening of $B$ molecules due to the polarizability anisotropy brings $E_{B}$ closer to $E_{A}$. The observed line shape of the $A$ molecule reveals a molecule-substrate interaction beyond image charge attraction: The broadening is comparable to the $\alpha$ phase and thus indicates wave function overlap between the $\pi$ system of the flat-lying molecule and the metal, leading to hybridization and lifetime broadening. In contrast, the spectra of all other molecules consist of sharp resonances, broadened only by additional satellite peaks due to vibronic progressions [24,25] at the high energy side of the main peak, and regions of negative differential conductance $[5,6,26]$.

We briefly rule out alternative explanations of the observed energy level dispersity: The LUMO energy shifts cannot be caused by a Stark effect, because we do not observe a dependence on the tip height, apart from a scatter $<30 \mathrm{meV}$ over a tip approach range of the order $1 \AA$. Also, a decisive influence of local electric fields originating from the charge distribution within molecule layers [27] can be excluded because we do not observe systematic changes of the energy level position with tip height. Since tetracene molecules are quite rigid and do not distort from their gas phase structure when condensing to form a bulk crystal [28], we eliminate molecular distortions as causes of energy level shifts. Finally, we rule out a significant influence of intermolecular charge delocalization on the screening in the tetracene $\beta$ phase, because in that case one would expect the lowest LUMO energy for $A$ and $C$ molecules which show the best $\pi$ stacking.

In conclusion, electronic spectra of individual molecules in the tetracene $\beta$ phase on $\operatorname{Ag}(111)$ reveal that molecular energy levels are not only subject to chemical shifts and polarization screening induced by the substrate, but also to site-specific polarization screening from neighboring molecules. Our data demonstrate that polarization screening in molecular crystals, being the convolution of a singlemolecule property (anisotropic polarizability) with the lattice structure of the film (many different environments due to complex unit cells and/or surfaces or interfaces), may lead to an electronic structure which varies strongly from molecule to molecule. In a certain sense, the materials thus can be considered as being made of electronically distinct species, and this should evidently have profound implications for their transport physics. The physical mechanism suggested in Fig. 4 may also explain the negative outcome of the attempts to measure surface core-level shifts for thin films of organic semiconductors $[12,13]$ : If the crystal structure of the films is such that the polarization screening of the photohole is mainly effected by molecules located in a plane parallel to the surface, surface core-level shifts are expected to be very small. In this sense, the present data reconcile the conflicting evidence with respect to polarization screening in Refs [11-13].

We thank M.B. Casu (University of Tübingen), M. Sokolowski (University of Bonn), A. Schöll and S. Sachs (Universität Würzburg), and N. Ueno (Chiba University) for helpful discussions.

*Corresponding author. s.tautz@fz-juelich.de

[1] J. Shinar, Organic Light-Emitting Devices: A Survey (Springer, New York, 2004).

[2] H. Klauk, Organic Electronics: Materials, Manufacturing, and Applications (Wiley-VCH, Weinheim, 2006).

[3] Physical and Chemical Aspects of Organic Electronics: From Fundamentals to Functioning Devices, edited by C. Wöll (Wiley-VCH, Weinheim, 2009).

[4] N. Ueno and S.Kera, Prog. Surf. Sci. 83, 490 (2008).

[5] M. Grobis et al., Appl. Phys. Lett. 86, 204102 (2005).

[6] K. J. Franke et al., Phys. Rev. Lett. 100, 036807 (2008).

[7] A. Hauschild et al., Phys. Rev. Lett. 94, 036106 (2005).

[8] Y. Zou et al., Surf. Sci. 600, 1240 (2006).

[9] L. Kilian et al., Phys. Rev. Lett. 100, 136103 (2008).

[10] E. A. Silinsh, Organic Molecular Crystals: Their Electronic States (Springer, Berlin, 1980).

[11] E. V. Tsiper et al., Chem. Phys. Lett. 360, 47 (2002).

[12] M. B. Casu, Phys. Status Solidi (RRL) 2, 40 (2008).

[13] M. B. Casu et al., Phys. Rev. B 76, 193311 (2007).

[14] R. Hesper, L. H. Tjeng, and G. A. Sawatzky, Europhys. Lett. 40, 177 (1997).

[15] A. Schöll et al., J. Phys. Chem. B 108, 14741 (2004).

[16] Y. Xia et al., Appl. Phys. Lett. 90, 162106 (2007).

[17] T. Takahashi et al., Adv. Funct. Mater. 17, 1623 (2007).

[18] A. Langner et al., Surf. Sci. 574, 153 (2005).

[19] Apparently, the $\beta$ phase is more complex than proposed in [18]. The $2.7 \AA$ tip retraction in going from the $\alpha$ to the $\beta$ phase [Fig. 1(b)], compared to $1.4 \AA$ for going from $\operatorname{Ag}(111)$ to $\alpha$, reveals that the $\beta$ phase is a bilayer. Note, however, that due to electronic structure effects, the latter number cannot be taken as an indicator of the actual step height.

[20] This dispersity is the reason why the layers of the $\beta$ phase can be imaged separately: The first layer $A$ and $B$ molecules in Fig. 2(a) are imaged through the energy gap of the second layer $C$ molecules.

[21] J. T. Li et al., Phys. Rev. Lett. 81, 4464 (1998).

[22] GAUSSIAN 03, M. J. Frisch et al., Gaussian, Inc., Pittsburgh PA, 2003.

[23] The calculation reveals that due to the larger nearestneighbor distance along the $x$ direction, screening in this direction is negligible, in spite of the highest polarizability in this direction.

[24] X. H. Qiu, G. V. Nazin, and W. Ho, Phys. Rev. Lett. 92, 206102 (2004).

[25] Th. Frederiksen et al., Phys. Rev. B 78, 233401 (2008).

[26] J. Repp et al., Phys. Rev. Lett. 94, 026803 (2005).

[27] S. Duhm et al., Nature Mater. 7, 326 (2008).

[28] K. Hummer and C. Ambrosch-Draxl, Phys. Rev. B 72, 205205 (2005). 